\title{
Overexpression of transforming growth factor- $\beta 1$ in fetal monkey lung results in prenatal pulmonary fibrosis
}

\author{
A.F. Tarantal*, H. Chen\#, T.T. Shi\#, C-H. Lu\#, A.B. Fang\#", S. Buckley\#, M. Kolb", \\ J. Gauldie ", D. Warburton ${ }^{\#}$ and W. Shi ${ }^{\#}$
}

ABSTRACT: Altered transforming growth factor (TGF)- $\beta$ expression levels have been linked to a variety of human respiratory diseases, including bronchopulmonary dysplasia and pulmonary fibrosis. However, a causative role for aberrant TGF- $\beta$ in neonatal lung diseases has not been defined in primates.

Exogenous and transient TGF- $\beta 1$ overexpression in fetal monkey lung was achieved by transabdominal ultrasound-guided fetal intrapulmonary injection of adenoviral vector expressing TGF- $\beta 1$ at the second or third trimester of pregnancy. The lungs were then harvested near term, and fixed for histology and immunohistochemistry.

Lung hypoplasia was observed where TGF- $\beta 1$ was overexpressed during the second trimester. The most clearly marked phenotype consisted of severe pulmonary and pleural fibrosis, which was independent of the gestational time point when TGF- $\beta 1$ was overexpressed. Increased cell proliferation, particularly in $\alpha$-smooth muscle actin-positive myofibroblasts, was detected within the fibrotic foci. But epithelium to mesenchyme transdifferentiation was not detected. Massive collagen fibres were deposited on the inner and outer sides of the pleural membrane, with an intact elastin layer in the middle. This induced fibrotic pathology persisted even after adenoviralmediated TGF- $\beta 1$ overexpression was no longer evident.

Therefore, overexpression of TGF- $\beta 1$ within developing fetal monkey lung results in severe and progressive fibrosis in lung parenchyma and pleural membrane, in addition to pulmonary hypoplasia.

KEYWORDS: Lung development, pulmonary fibrosis, transforming growth factor- $\beta$

ung development, including early branching morphogenesis and alveolarisation, are well conserved among human and nonhuman primate species [1]. Thus, the nonhuman primate provides an excellent model for studying lung development and the mechanism(s) associated with organogenesis, as well as the pathology of congenital respiratory diseases in humans [2]. Lung development has been divided morphologically into the embryonic stage (week 4-6 of human pregnancy, gestation day 40-59 in monkeys with a full-term pregnancy $165 \pm 10$ days); the pseudoglandular stage (week 6-17 of human pregnancy, gestation day 59-82 in monkeys); the canalicular stage (week 16-25 of human pregnancy, gestation day 82-140 in monkeys); the saccular stage (week 24-36 in human pregnancy, gestation day 120-150 in monkeys); and the alveolar stage (week 36 of human pregnancy to 7 yrs postnatal, near term to 3 yrs postnatal in monkeys) [1]. The canalicular stage encompasses a transformation to a potentially 'viable' lung, with development of the future gas exchange area, increasing vascularisation of the mesenchyme surrounding the airways, and emergence of recognisably mature distal lung epithelial cells with the capability to produce surfactant proteins. Lung development is regulated by many factors, including transforming growth factor (TGF)- $\beta$ [3]

The TGF- $\beta$ family comprises a group of growth factors that regulate many fundamental biological processes [4-6]. TGF- $\beta$ signalling plays an important role in lung development, injury repair and remodelling, as well as pulmonary inflammatory responses [7]. Alteration of TGF- $\beta$ signalling may

\section{AFFILIATIONS}

${ }^{*}$ Center for Fetal Monkey Gene Transfer for Heart, Lung, and Blood Diseases, California National Primate Research Center and Depts of Pediatrics and Cell Biology and Human Anatomy, School of Medicine, University of California, Davis, and

"Developmental Biology and Regenerative Medicine Program Childrens Hospital Los Angeles, 4650 Sunset Blvd, Los Angeles, CA USA.

"Dept of Medicine, Pathology and Molecular Medicine, McMaster University, Hamilton, ON, Canada.

\section{CORRESPONDENCE}

W. Shi

Developmental Biology and Regenerative Medicine Program, Dept of Surgery

Childrens Hospital Los Angeles 4650 Sunset Blvd

MS 35

Los Angeles

CA 90027

USA

E-mail: wshi@chla.usc.edu

Received:

Jan 222010

Accepted after revision:

March 062010

First published online:

March 292010 
be associated with bronchopulmonary dysplasia (BPD), severity of cystic fibrosis, asthma, idiopathic pulmonary fibrosis and emphysema in humans, depending on the developmental age of onset of the specific condition [8-13]. Overexpression of TGF- $\beta 1$ driven by a $3.7 \mathrm{~kb}$ human surfactant protein (SP)-C promoter into fetal lung epithelium of transgenic mice resulted in hypoplastic fetal lung phenotypes [14]. Moreover, selective abrogation of TGF- $\beta$ signalling in developing mouse lung epithelial cells and mesenchymal cells of TGF- $\beta$ receptor II conditional knockout mice also resulted in retarded postnatal lung alveolarisation and abnormal fetal lung branching morphogenesis, respectively [15]. Taken together, these gain and loss of function findings suggest that tightly regulated TGF- $\beta$ signalling activity plays an essential role in regulating lung development and function in rodents. However, the role of TGF- $\beta$ signalling in regulating human and nonhuman primate fetal lung development is still not completely clear. In this study we have addressed the pathogenic role of excessive focal activation of TGF- $\beta$ signalling in fetal rhesus monkey lung, and have shown that overexpression of active TGF- $\beta$ at defined developmental stages results in severe and progressive fibrosis, particularly in the pleural membrane.

\section{MATERIALS AND METHODS Adenoviral vector preparation}

An adenoviral vector that expressed biologically active human TGF- $\beta 1$ (Ad-TGF- $\beta^{223 / 225}$ ) was prepared as published previously [16]. The same adenoviral vector-mediated gene expression in adult rodent lung persisted for about 10 days in vivo [16].

\section{Animals}

All animal procedures conformed to the requirements of the Animal Welfare Act and protocols were approved prior to implementation by the institutional animal care and use committee at the University of California, Davis, CA, USA. Normally cycling, adult female rhesus monkeys (Macaca mulatta; $\mathrm{n}=6$ ) with a history of prior pregnancy were bred and identified as pregnant using established methods [17]. Pregnancy in the rhesus monkey is divided into trimesters by 55-day increments, with 0-55 days gestation representing the first trimester, 56-110 days gestation representing the second trimester, and 111-165 days gestation the third trimester (term $165 \pm 10$ days) [18].

\section{Vector administration and fetal monitoring}

All fetuses were sonographically assessed to confirm normal growth and development prior to gene transfer [17]. The dams were administered ketamine hydrochloride $\left(10 \mathrm{mg} \cdot \mathrm{kg}^{-1} i . m\right.$.) or telazol (5-8 $\mathrm{mg} \cdot \mathrm{kg}^{-1} i . m$.) for ultrasound examinations. On the day of gene transfer, the dams were administered telazol and aseptically prepared for transabdominal ultrasound-guided fetal intrapulmonary gene delivery [17, 18]. Approximately $100 \mu \mathrm{L}$ of the Ad-TGF- $\beta^{223 / 225}$ was injected into the right or left lung lobes under ultrasound guidance in the late second trimester ( $~ 100$ days gestation; $n=3$ ) or third trimester ( $\sim 140$ days gestation; $\mathrm{n}=2$ ) using an intrapulmonary approach as previously reported [19]. One control animal received the Ad-EGFP vector at $\sim 100$ days gestation, in which a cDNA encoding EGFP was used to replace TGF- $\beta^{223 / 225}$ CDNA of the same adenoviral vector. Post-gene transfer, sonographic measurements of the fetal head and limbs, in addition to gross anatomical evaluations, were assessed during gestation, as previously described [17].

\section{Tissue harvest}

The one animal that received the adenoviral EGFP vector was harvested 4 days post-gene transfer to verify adenoviralmediated gene expression by detecting EGFP (fig. 1a). A side-by-side comparison of comparable tissues from control animals was performed to address the potential for autofluorescence (not shown) [19].

All five fetuses that received the Ad-TGF- $\beta^{223 / 225}$ vector and two control fetuses were harvested near term (gestation day $150 \pm 2$ ) using established protocols [19]. Total body weights and measures (hand, foot, humerus and femur lengths, biparietal and occipitofrontal diameters, head, arm and chest circumferences, and crown-rump lengths) were assessed, then all organs were removed and weighed after carefully examining the thoracic and abdominal cavities, and multiple tissues were collected, including all lung lobes. Caudal lung lobes were cannulated and fixed with $2 \%$ paraformaldehyde at $30 \mathrm{cmH}_{2} \mathrm{O}$ for $4 \mathrm{~h}$ [19]. The total volumes of the fixed lobes were determined by volume displacement using established techniques. Each of the caudal lung lobes was then cut into sections perpendicular to the primary axial path of the lobar bronchus. Low magnification images of the six cut surfaces of each of the caudal lobe slices were digitally captured using a Leica dissecting microscope and analysed with a point counting grid at low magnification (fig. 1b). The embedded lung tissues were then sectioned at $5 \mu \mathrm{m}$, and stained with haematoxylin and eosin (H\&E) for routine histology. Specimens from animals of comparable age without any interventions (controls) were similarly processed and analysed in parallel.

\section{Immunohistochemistry}

The following antibodies were used in these studies: SP-C rabbit polyclonal antibody (Santa Cruz Biotechnology Inc., Santa Cruz, CA, USA), $\alpha$-smooth muscle actin mouse monoclonal antibody (Sigma, St Louis, MO, USA), and E-cadherin rabbit monoclonal antibody (Epitomics, Burlingame, CA, USA). HistoStain kit from Invitrogen (South San Francisco, CA, USA) was used. For double immunofluorescence staining, lung sections were incubated with primary antibodies overnight at $4^{\circ} \mathrm{C}$, followed by donkey secondary antibodies conjugated with fluorescence (Alexa Fluor-594 or Alexa Fluor-488; Invitrogen). Cell proliferation was analysed by immunostaining proliferating cell markers Ki67 (Thermo, Rockford, IL, USA) and proliferating cell nuclear antigen (PCNA; Invitrogen). Normal serum or immunoglobulin G was used to replace the primary antibody as negative control.

\section{Sirius red collagen staining}

The rehydrated lung tissue sections were first stained with Weigert's haematoxylin for cell nuclei. After washing in running tap water for $10 \mathrm{~min}$, the sections were then stained with Sirius red $(0.1 \%$ in saturated picric acid) for $1 \mathrm{~h}$, followed by washing in two changes of acidified water $(0.5 \%$ acetic acid in water). The mounted slides were examined under a light microscope (Carl Zeiss, Goettingen, Germany) with crossed polar filters. 


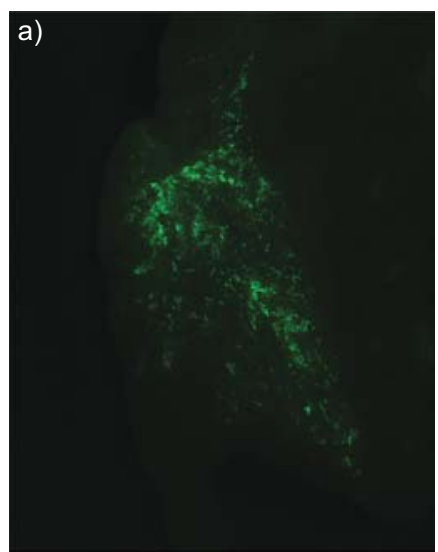

b)
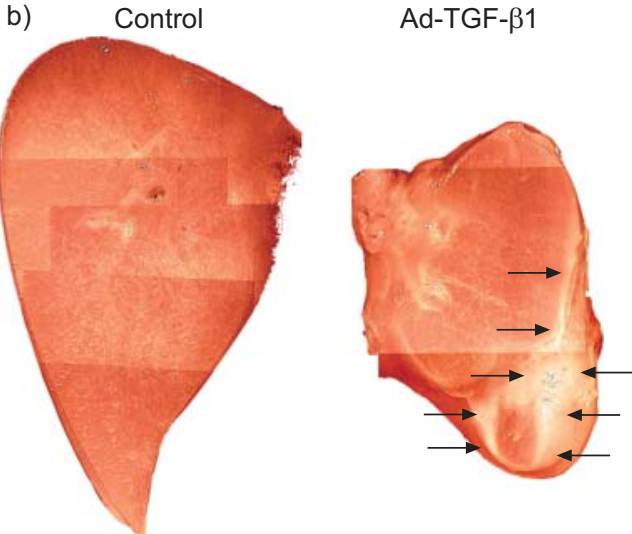

d)

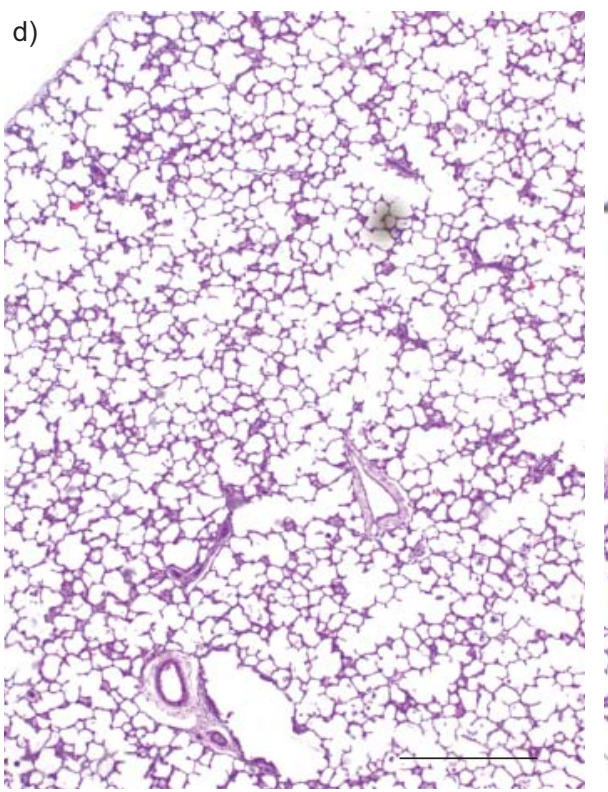

e)

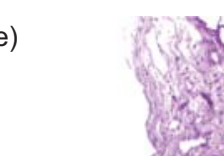

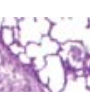
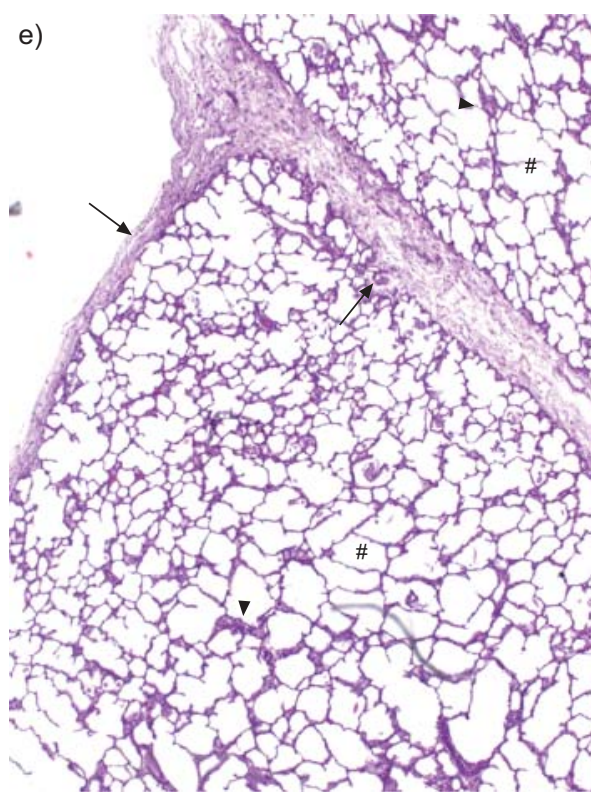
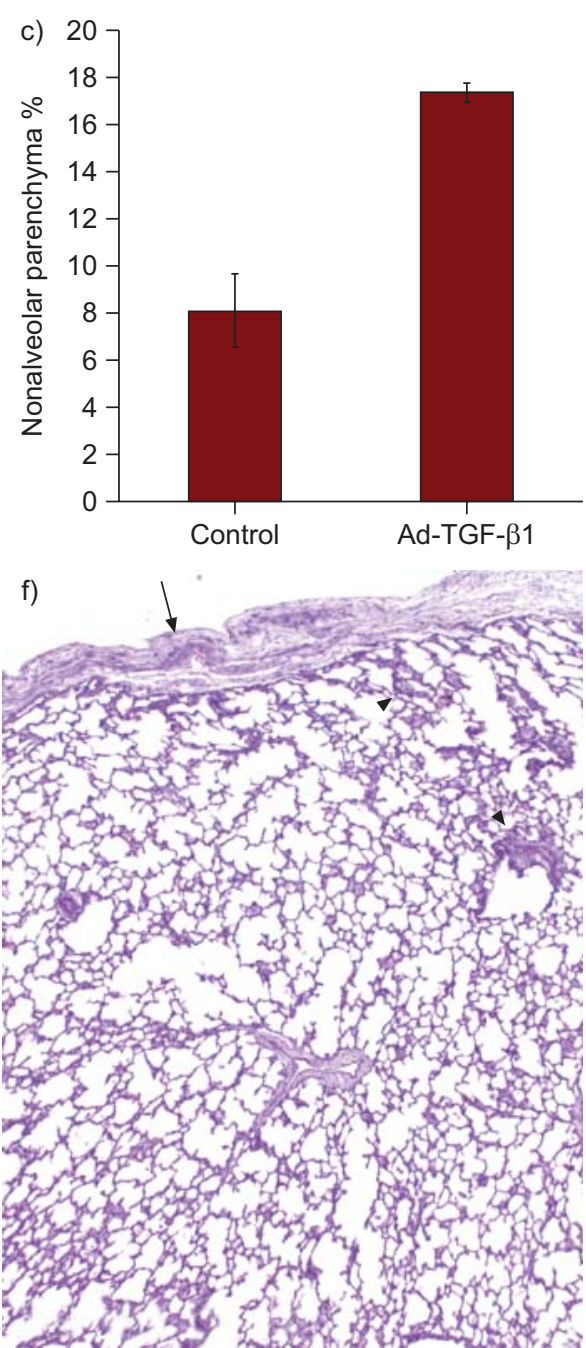

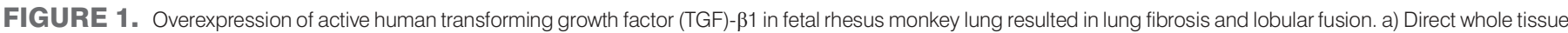

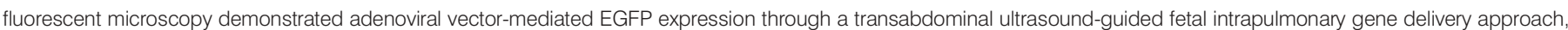

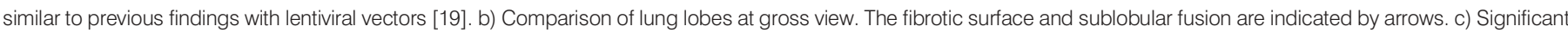

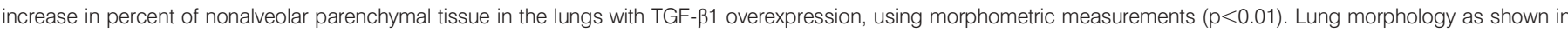

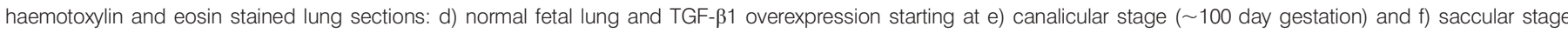
( $\sim 140$ day gestation). Fibrotic pleural membranes (arrows), lung interstitial fibrosis (arrowheads) and focal mild enlargement of alveoli (\#) are indicated. Scale bar $=500 \mu$ m.

\section{Elastin staining}

Elastin was stained using Hart's resorcin-fuchsin solution as published previously [15].

\section{Statistical analysis}

Statistical significance $(\mathrm{p}<0.05)$ was determined by ANOVA or two-sided unpaired t-test analysis.

\section{RESULTS}

As shown in rodents, the TGF- $\beta$ ligand and its intracellular signalling play important regulatory roles during lung development and after tissue injury repair [15, 20]. However, whether alteration of TGF- $\beta 1$ activity in developing nonhuman primate fetal lung results in similar pathological changes has not, to our knowledge, been studied. In these studies we used an adenoviral vector-mediated gene transfer approach to overexpress exogenous TGF- $\beta 1$ transiently in fetal rhesus monkey lung in the second or third trimesters (canalicular stage Ad-TGF- $\beta 1$ (C), 100 days gestation; or saccular stage Ad-TGF- $\beta 1$ (S), $\sim 140$ days gestation) and harvested tissues near term (alveolar stage, $150 \pm 2$ days gestation).

\section{Fetal growth and development}

Sonographic measurements of the fetal head, abdomen and limbs, in addition to anatomical evaluations were assessed every 7-10 days post-gene transfer, as previously described [17]. All parameters evaluated were compared to normative growth charts for rhesus fetuses and found to be within normal limits (data not shown). Lobes in three lungs with TGF- $\beta 1$ overexpression (one Ad-TGF- $\beta 1$ (C) and two Ad-TGF- $\beta 1$ (S)) were partially fused, and covered by a thick connective tissue membrane (fig. 1b), while lobes on the contralateral (uninjected) side appeared normal. These fused lung lobes had a substantially lower percent of alveolar parenchyma when compared to controls $(\mathrm{p}<0.01)$ (fig. 1c). The lung lobe weights were highly variable due to degree of fusion (not shown). 


\section{Overexpression of TGF- $\beta 1$ resulted in fetal lung fibrosis}

Lung morphology was further examined in H\&E stained lung tissue sections. Overexpression of human biologically active TGF- $\beta 1$ ligand in the early canalicular stage (Ad-TGF- $\beta 1$ (C)) caused focal and relatively mild terminal air sac enlargement (fig. 1d-f). However, marked diffuse interstitial pulmonary fibrosis of the alveolar septa, the pulmonary interstitial reticulum and, most strikingly, the pleural membranes comprised the major lung pathologies (fig. 1d-f). Similarly, marked pulmonary fibrotic pathology was also observed in the lung with TGF- $\beta 1$ overexpression at later gestation (Ad-TGF- $\beta 1$ (S)), while focal and significant changes in alveolar size were not observed.

Interestingly, overexpression of TGF- $\beta 1$ at both gestational stages (canalicular stage versus saccular stage) resulted in severe fibrosis in the pleural membranes, and fusion and obliteration of interlobular pleural clefts (fig. 1d-f). These fibrotic foci were further extended into adjacent lung parenchymal tissues. In addition, fibrotic pathology was also observed in some perivascular tissues as well as bronchiolar conducting airway walls. It is important to note that the control animal, which received the adenoviral vector with EGFP only, was grossly and histologically normal. Thus, the specific lung pathology observed is likely a result of TGF- $\beta 1$ overexpression at critical developmental time points.

\section{Overexpression of TGF- $\beta 1$ resulted in increased cell proliferation within fibrotic loci}

Previous studies in vivo and in vitro have suggested that TGF- $\beta$ has complex regulatory effects on cell growth, which are dependent on the cell type and developmental stage. Thus, we examined changes in cell proliferation of the lung with early TGF- $\beta 1$ overexpression. As detected by Ki67 immunostaining, cell proliferation was significantly increased in lung septa and pleural membranes, particularly within fibrotic loci of lungs that received Ad-TGF- $\beta 1$ vector injection at either the canalicular stage or the saccular stage (fig. 2). Most Ki67positive cells were localised to the pleural membrane and mesenchymal compartment, where progressive lung fibrosis developed shortly after Ad-TGF- $\beta 1$ gene transfer (fig. 2). Interestingly, increased Ki67-positive cells, as compared to normal controls, were still detected in lung septal structures even after $>50$ days post-adenoviral vector infection (fig. 2), when exogenous TGF- $\beta 1$ expression should clearly no longer be present. Proliferative changes were confirmed using an antibody recognising another proliferating cell marker, PCNA (data not shown). These findings suggest that overexpression of TGF- $\beta 1$ may trigger increased cell proliferation, particularly in mesenchymal cells. These activated cells then continue to proliferate in a TGF- $\beta$-independent manner well after the overexpression of exogenous TGF- $\beta 1$ has been discontinued.

\section{Lung epithelial cells versus mesenchymal cells}

We further determined changes in the lung cell types following exogenous TGF- $\beta 1$ overexpression. Interestingly, SP-C-positive cells were significantly increased in the area adjacent to the fibrotic pleural membranes caused by TGF- $\beta 1$ overexpression (fig. 3), particularly in the lung with relatively recent exogenous TGF- $\beta 1$ gene transfer ( 2 weeks after Ad-TGF- $\beta 1$ was injected). Moreover, $\alpha$-smooth muscle actin-positive cells were also increased in the lung with TGF- $\beta 1$ overexpression. Most of the $\alpha$-smooth muscle actin-positive cells were found within the walls of vessels adjacent to fibrotic pleural membranes. Increased myofibroblasts were also observed in subpleural interstitial tissues, even though exogenous TGF- $\beta 1$ was expressed earlier at the canalicular stage. This suggests that while TGF- $\beta 1$ may trigger ongoing proliferation of these cells, subsequent cell population expansion may be TGF- $\beta$ independent. We further looked into whether there were a significant number of cells that transdifferentiated from epithelial cells to mesenchymal cells. By co-immunostaining with epithelial cell marker E-cadherin and
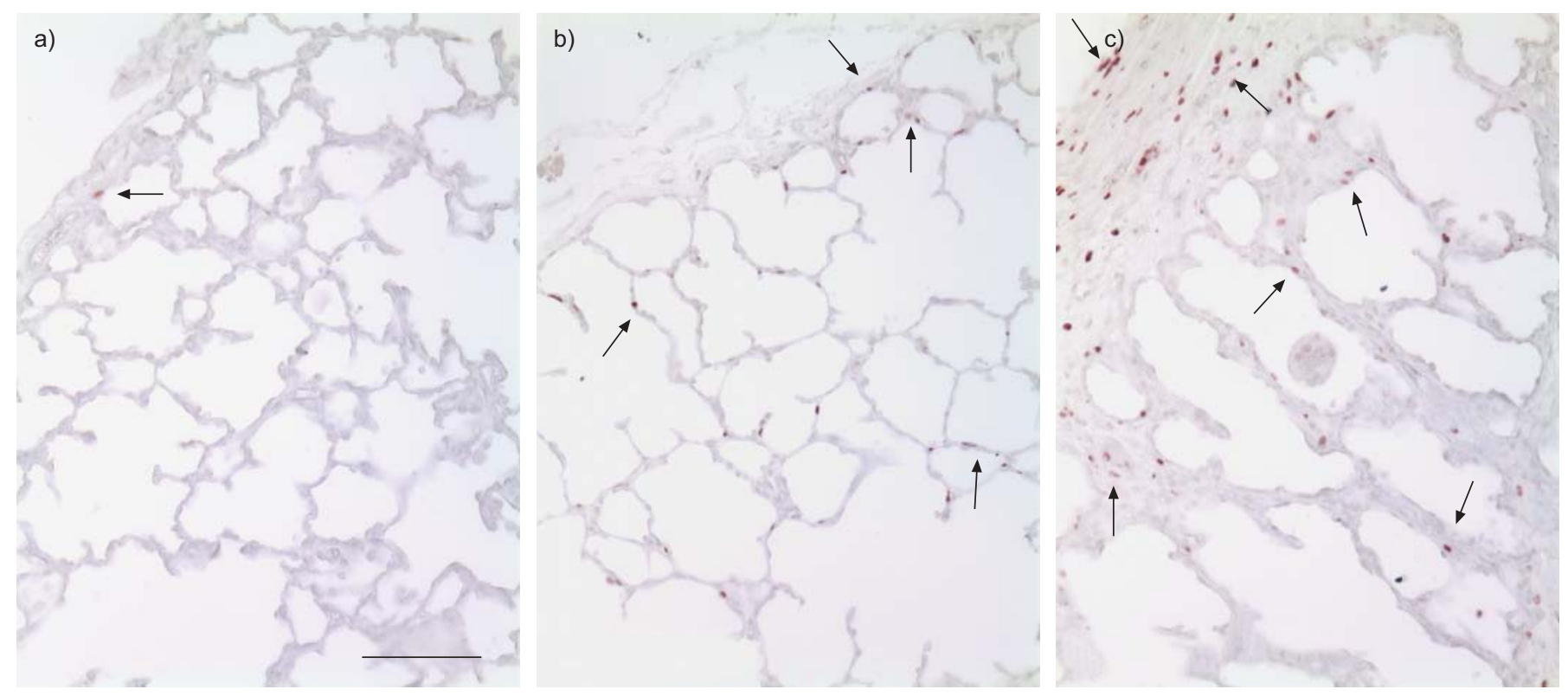

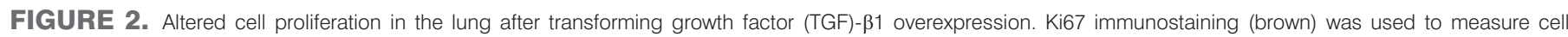

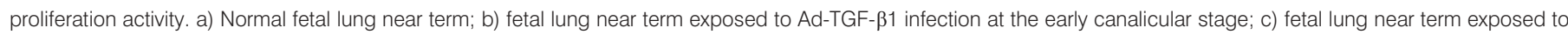
Ad-TGF- $\beta 1$ in the saccular stage. Scale bar $=100 \mu \mathrm{m}$. 


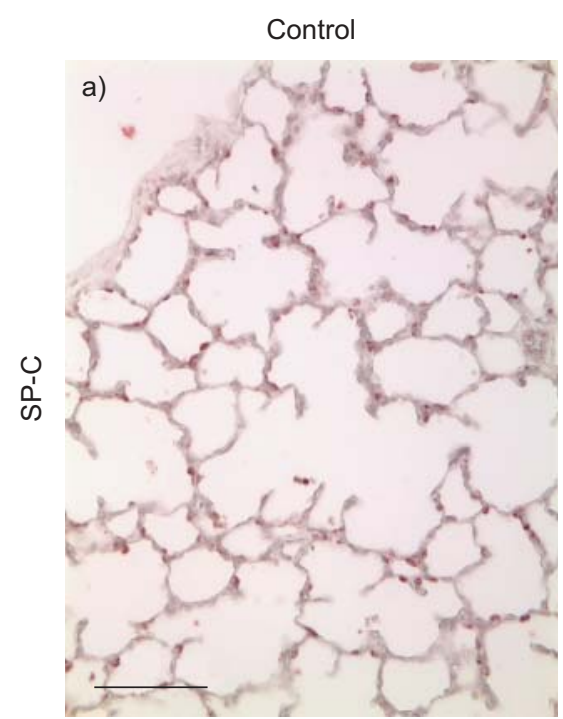

d)
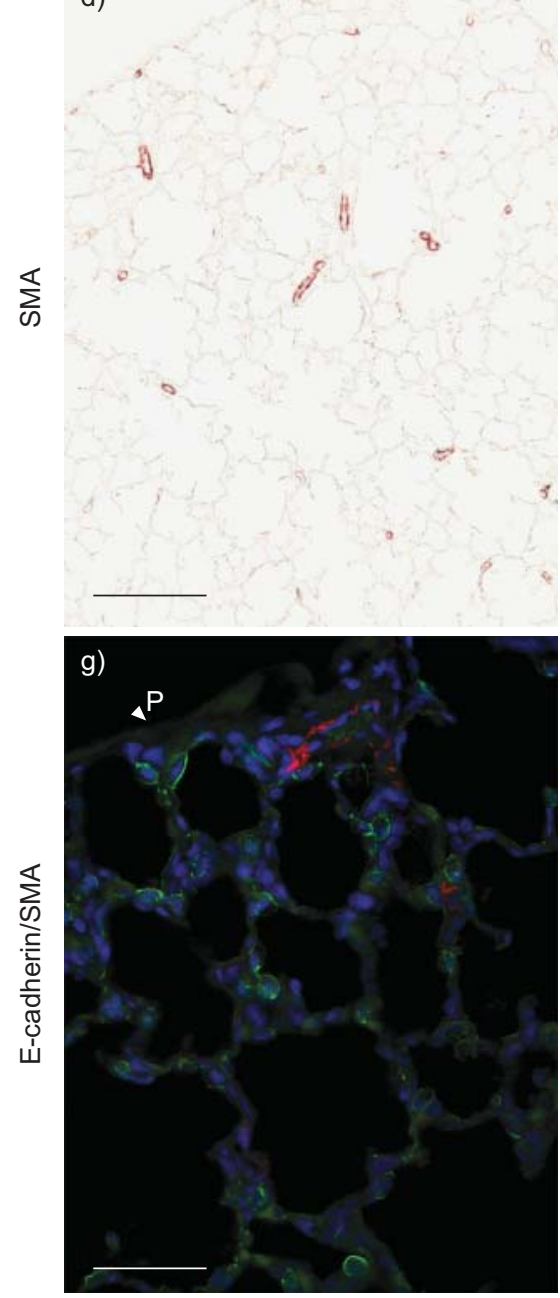

$\operatorname{Ad-TGF\beta 1~(C)~}$

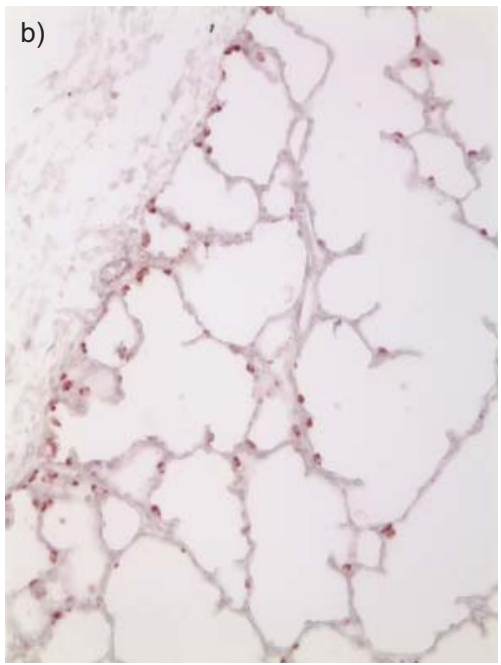

e)
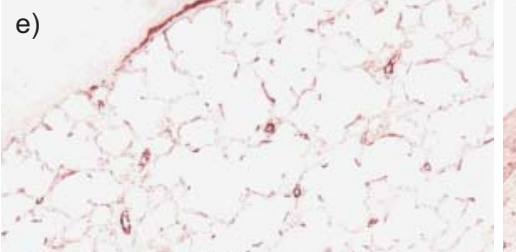

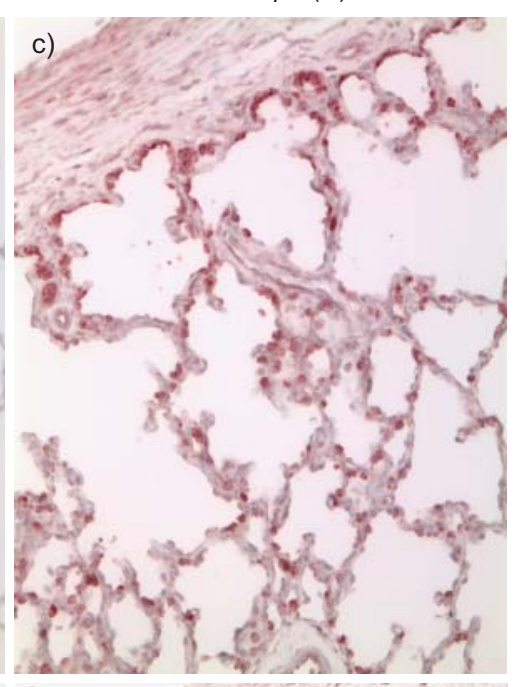

f)
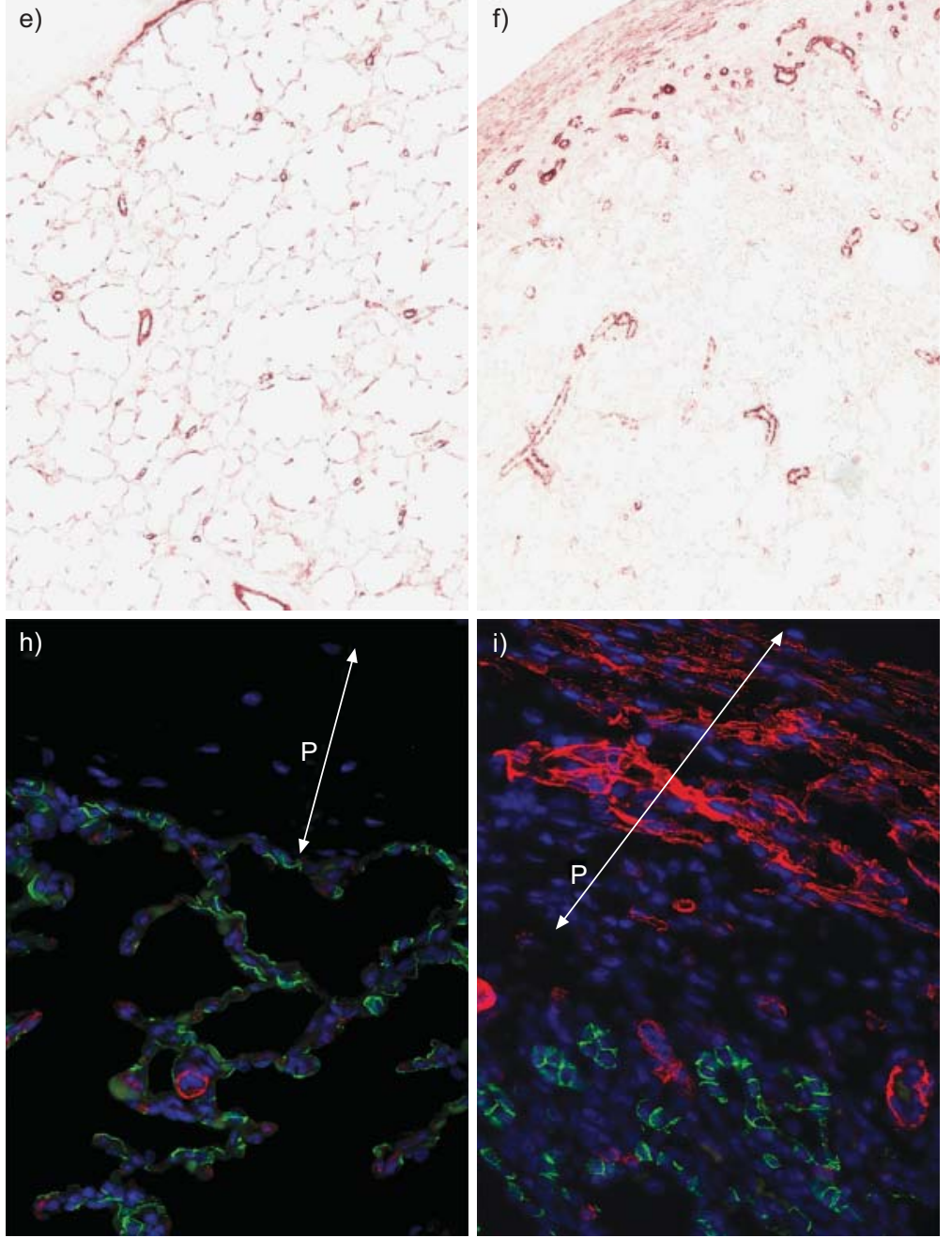

FIGURE 3. Changes in peripheral lung epithelial and mesenchymal cells following transforming growth factor (TGF)- $\beta 1$ overexpression. Type II alveolar epithelial cells and myofibroblasts were detected by surfactant protein (SP)-C (red) and $\alpha$-smooth muscle actin (SMA; red) immunostaining, respectively. Furthermore, coimmunofluorescence staining for E-cadherin (green) and SMA (red) was performed. Cell nuclei were counterstained with 4',6-diamidino-2-phenylindole (blue). Pleural membrane $(P)$ is indicated by arrows. a) Scale bar $=100 \mu \mathrm{m}$; d) scale bar=200 $\mu \mathrm{m} ; \mathrm{g})$ scale bar $=50 \mu \mathrm{m}$. 
myofibroblast marker $\alpha$-smooth muscle actin, cells with overlapping fluorescence signals were barely detected in these lung specimens (fig. 3), suggesting that epithelial-to-mesenchymal transdifferentiation was not occurring at those time points. Thus, it is less likely that epithelium-to-mesenchyme transdifferentiation significantly contribute to the related fibrotic pathology in this case. In addition, significantly increased myofibroblasts were detected in pleural membrane in Ad-TGF- $\beta 1$ (S) lung, but not in Ad-TGF- $\beta 1$ (C) lung (fig. 3). This suggests that increased TGF- $\beta 1$ initiates pleural fibrosis by activating myofibroblasts during TGF- $\beta 1$ overexpression, while the deposited fibrotic tissues in pleural membrane do not resolve even after cessation of TGF- $\beta 1$ overexpression.

\section{Altered extracellular collagen and elastin deposition and structures}

TGF- $\beta$ is a well-known growth factor that stimulates extracellular matrix protein production, including collagen and elastin. We therefore compared these extracellular proteins in lung tissues with TGF- $\beta 1$ overexpression versus controls. Consistent with H\&E-stained lung morphology, a large quantity of collagen fibres were produced and deposited in pleural membranes with TGF- $\beta 1$ overexpression, giving the appearance of "bread and butter" pleura (fig. 4). In addition, increased and thickened collagen fibres were also noted interspersed within septal structures, with some of these fibres connected through the pulmonary interstitial reticulum to the fibrotic pleural membrane. Interestingly, thickened elastin fibres also appeared to be localised within fibrotic foci, and an intact elastin fibre layer was also detected within the fibrotic pleural membrane, with excessive collagen deposition on both the inner and outer sides (fig. 4).

\section{DISCUSSION}

Data from both in vitro and in vivo studies suggest that TGF- $\beta$ has compound biological activities in regulating cell proliferation,
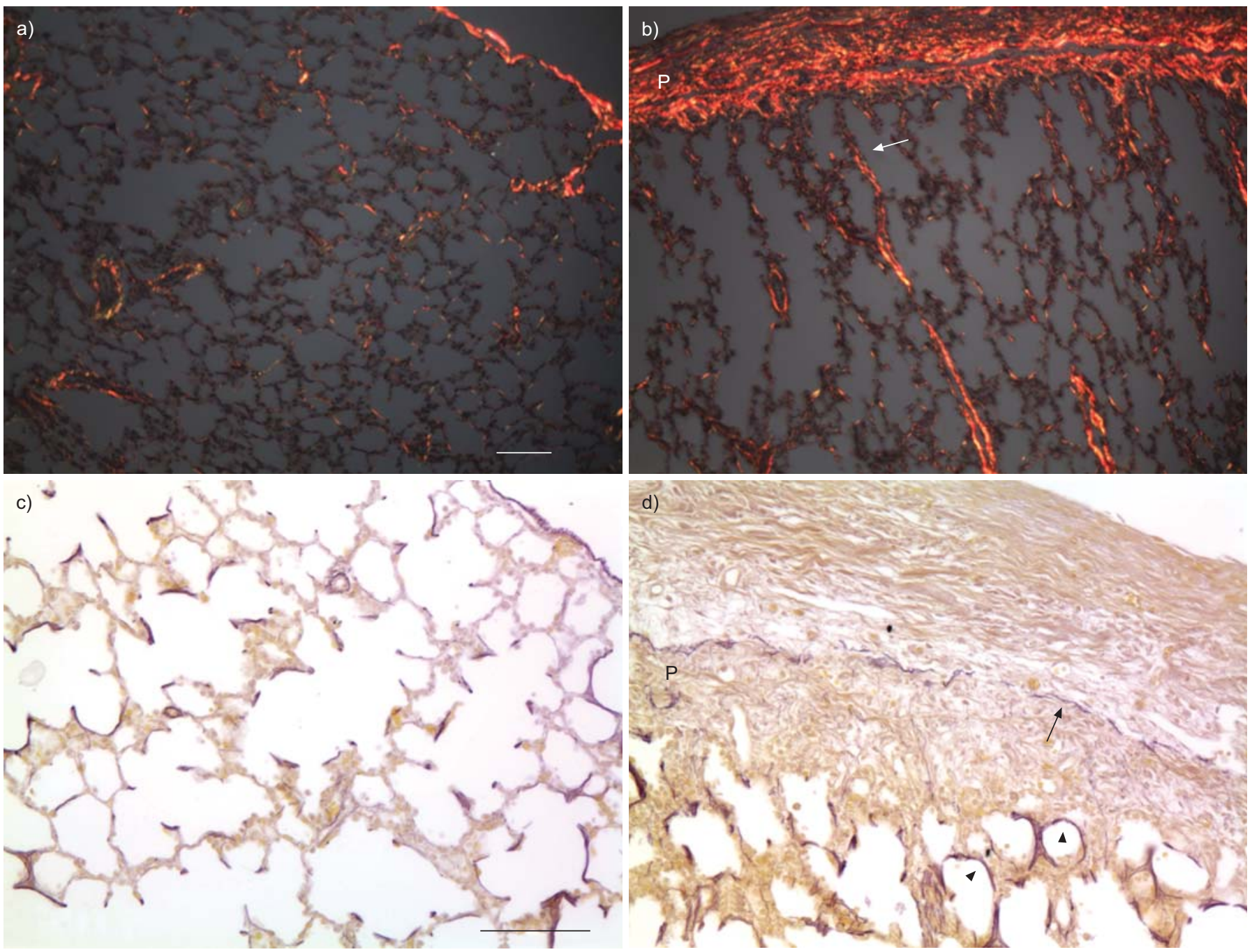

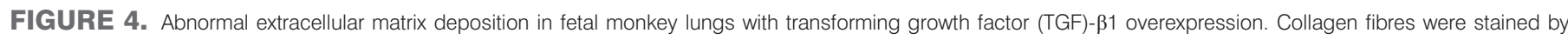

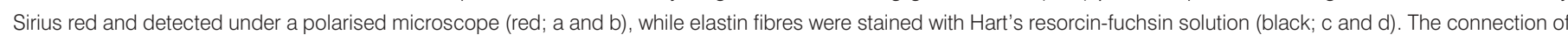

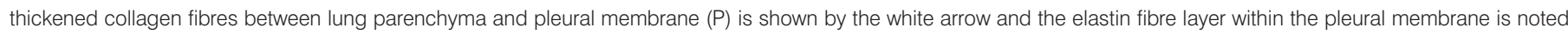

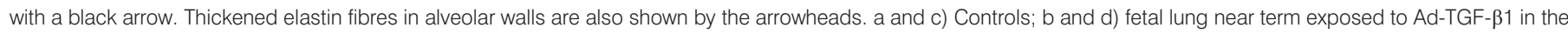
saccular stage. Scale bars $=100 \mu \mathrm{m}$. 
differentiation, and extracellular matrix production, which are dependent on the cell type and status, as well as the duration and levels of TGF- $\beta$ stimulation. Studies focused on TGF- $\beta$ signalling in lung development and related neonatal respiratory diseases also provide some contradictory results. For example, in ex vivo early mouse embryonic lung (E11.5) explant culture, excess TGF- $\beta 1$, either by directly adding exogenous TGF- $\beta 1$ ligand to the culture medium or by adenoviral vector-mediated TGF- $\beta 1$ overexpression, significantly inhibits lung branching morphogenesis during the pseudoglandular stage [21, 22]. Similarly, constant transgenic overexpression of TGF- $\beta 1$ in mouse developing lung epithelial cells results in hypoplastic lung and neonatal lethality [14]. However, fibrotic pathology in fetal mouse lungs was not observed in these studies. Interestingly, abrogation of endogenous TGF- $\beta$ signalling in developing mouse lung epithelial cells or mesenchymal cells did not cause a predicted converse lung phenotype compared to TGF- $\beta$ overexpression, e.g. hyperplastic branching [15]. Moreover, both increased and decreased TGF- $\beta$ levels have been detected in BPD patients $[23,24]$, in whom normal lung development is disrupted. Therefore, in order to further understand the relationship between altered levels of TGF- $\beta$ and lung development/ maturation, we have studied the biological effects of transient overexpression of TGF- $\beta 1$ on nonhuman primate fetal lung development, an animal model that closely simulates human lung development. In contrast to findings in rodents, the major pulmonary phenotype seen after overexpression of TGF- $\beta 1$ in the second or third trimesters in fetal rhesus monkey lung was fibrosis.

Excess TGF- $\beta$ signal pathway activation in the lung from adenoviral vector-mediated overexpression of biologically active TGF- $\beta 1$ ligand has previously been reported to induce chronic, aggressive fibrosis in adult rats [16]. Increased expression of TGF- $\beta 1$ has also been reported in adults with interstitial pulmonary fibrosis. However, in the human neonate, increased expression of TGF- $\beta 1$ in bronchoalveolar fluid aspirates has been shown to be associated with adverse outcomes of BPD, a disease that is currently characterised by alveolar hypoplasia, rather than interstitial fibrosis per se. Thus, it is of interest to determine whether there would be a causative relationship between prenatal exposure to excessive TGF- $\beta 1$ activity, mediated by adenoviral vector expression, and abnormal lung formation in the fetal primate lung. The results of the current study indicate that the major outcome is one of ongoing, severe and progressive fibrosis that continues after cessation of TGF- $\beta 1$ expression, although a moderate hypoplastic lung phenotype is also detected, but only in subjects with earlier TGF- $\beta 1$ overexpression at the canalicular stage. This suggests that fetal primate lung behaves more like adult rodent lung than human lungs of extremely premature infants cared for in the modern era of neonatology. However, prior to the modern definition of BPD, the classical BPD phenotype in premature infants was comprised of emphysematous changes with interstitial lung fibrosis [25], while another classical chronic lung disease of prematurity, termed Wilson-Mikity disease, was characterised by pulmonary fibrosis [26].

A striking feature of the fibrotic pathology shown in the current study is extreme pleural thickening and fibrosis, which appears similar to what has been termed by classical pathologists "bread and butter" pleura. Nevertheless, this fibrosis was restricted to the visceral pleura, suggesting that it may not be mediated by soluble factors capable of crossing the pleural cavity to the parietal pleura. Furthermore, the question is raised of how fibrosis engendered by Ad-TGF- $\beta 1$ expression in relatively restricted areas at the site of vector injection located deep within the lung can be propagated all the way to the pleura, a finding which was also noted in rat models of AdTGF- $\beta 1$-induced fibrosis [16]. Based on the histological findings in the present study, it was noted that strands of thickened pulmonary interstitial reticulum extended from deep within the lung to the pleura. This is a greater distance than active TGF- $\beta 1$ produced by the transferred adenoviral vector could possibly diffuse. On the other hand, QuE et al. [27] reported that $\sim 30 \%$ of the smooth muscle cells within the pulmonary vascular walls, as well as some mesenchymal cells in alveolar septa, were derived from mesothelial cells in pleural membrane during early embryonic mouse lung development. Therefore, we postulate that the signal may be propagated in some fashion from cell to cell, or by attracting activated myofibroblast cell migration along the length of the reticulum in both directions.

In addition, our above results also indicate that a simple replenishment of active TGF- $\beta 1$ factor as a potential therapeutic approach for patients with deficient TGF- $\beta$ signalling in the lung during fetal development may not be beneficial as it has strong and diffusible pulmonary fibrogenic potency even in fetuses. However, this study demonstrates the feasibility of studying the effects of growth factor misexpression in the nonhuman primate lung, and the importance of this primate model system for the study of human fetal lung health and disease.

\section{SUPPORT STATEMENT}

These studies were supported by the National Heart, Lung, and Blood Institute (NIH; Bethesda, MD, USA) Center for Fetal Monkey Gene Transfer for Heart, Lung, and Blood Diseases (grant \#HL069748), the Primate Center base operating grant (\#RR00169), other National Institutes of Health support (grants \#HL068597, W. Shi; \#HL60231, \#HL44060 and \#HL44977, D. Warburton), California Institute of Regenerative Medicine training grant (D. Warburton), and the Webb Foundation at Children's Hospital Los Angeles, CA, USA (W. Shi). T.T. Shi, C-H. Lu and A.B. Fang are summer students.

\section{STATEMENT OF INTEREST}

None declared.

\section{REFERENCES}

1 Warburton D, Schwarz M, Tefft D, et al. The molecular basis of lung morphogenesis. Mech Dev 2000; 92: 55-81.

2 Fannuchi MV, Plopper CG. Pulmonary developmental responses to toxicants. In: Sipes IG, McQueen CA, Gandolfi AJ, eds. Comprehensive Toxicology, Volume 8: Toxicology of the Respiratory System. New York, Elsevier Science Ltd, 1997; pp. 203-220.

3 Warburton D, Bellusci S, Del Moral PM, et al. Cell signaling and growth factors in lung development. In: Unsicker K, Krieglstein K, eds. Cell Signaling and Growth Factors in Development, From Molecules to Organogenesis. Weinheim, Wiley-VCH, 2006; pp. 791-815. 
4 Sporn $\mathrm{MB}$, Roberts $\mathrm{AB}$. The transforming growth factor $\beta \mathrm{s}$. In: Sporn MB, Roberts AB, eds. Peptide Growth Factors and their Receptor: Handbook of Experimental Pharmacology. Heidelberg, Springer-Verlag, 1991; pp. 419-472.

5 Shi Y, Massague J. Mechanisms of TGF-beta signaling from cell membrane to the nucleus. Cell 2003; 113: 685-700.

6 Attisano L, Wrana JL. Smads as transcriptional co-modulators. Curr Opin Cell Biol 2000; 12: 235-243.

7 Warburton D, Bellusci S, Del Moral PM, et al. Growth factor signaling in lung morphogenetic centers: automaticity, stereotypy and symmetry. Respir Res 2003; 4: 5.

8 Khalil N, Parekh TV, O'Connor R, et al. Regulation of the effects of TGF-beta 1 by activation of latent TGF-beta 1 and differential expression of TGF-beta receptors (T beta R-I and T beta R-II) in idiopathic pulmonary fibrosis. Thorax 2001; 56: 907-915.

9 Toti P, Buonocore G, Tanganelli P, et al. Bronchopulmonary dysplasia of the premature baby: an immunohistochemical study. Pediatr Pulmonol 1997; 24: 22-28.

10 Drumm ML, Konstan MW, Schluchter MD, et al. Genetic modifiers of lung disease in cystic fibrosis. N Engl J Med 2005; 353: 1443-1453.

11 Celedon JC, Lange C, Raby BA, et al. The transforming growth factor-beta1 (TGFB1) gene is associated with chronic obstructive pulmonary disease (COPD). Hum Mol Genet 2004; 13: 1649-1656.

12 Pulleyn LJ, Newton R, Adcock IM, et al. TGFbeta1 allele association with asthma severity. Hum Genet 2001; 109: 623-627.

$13 \mathrm{Wu}$ L, Chau J, Young RP, et al. Transforming growth factor-beta1 genotype and susceptibility to chronic obstructive pulmonary disease. Thorax 2004; 59: 126-129.

14 Zhou L, Dey CR, Wert SE, et al. Arrested lung morphogenesis in transgenic mice bearing an SP-C-TGF-beta 1 chimeric gene. Dev Biol 1996; 175: 227-238.

15 Chen H, Zhuang F, Liu YH, et al. TGF- $\beta$ receptor II in epithelia versus mesenchyme plays distinct role in developing lung. Eur Respir J 2008; 32: 285-295.

16 Sime PJ, Xing Z, Graham FL, et al. Adenovector-mediated gene transfer of active transforming growth factor-beta1 induces prolonged severe fibrosis in rat lung. J Clin Invest 1997; 100: 768-776.
17 Tarantal AF. Ultrasound imaging in rhesus (Macaca mulatta) and long-tailed (Macaca fascicularis) macaques: Reproductive and research applications. In: Wolfe-Coote $\mathrm{S}$, ed. The Laboratory Primate. San Diego, Elsevier Academic Press, 2005; pp. 317-352.

18 Tarantal AF, Gargosky SE. Characterization of the insulin-like growth factor (IGF) axis in the serum of maternal and fetal macaques (Macaca mulatta and Macaca fascicularis). Growth Regul 1995; 5: 190-198.

19 Tarantal AF, McDonald RJ, Jimenez DF, et al. Intrapulmonary and intramyocardial gene transfer in rhesus monkeys (Macaca mulatta): safety and efficiency of HIV-1-derived lentiviral vectors for fetal gene delivery. Mol Ther 2005; 12: 87-98.

20 Gauldie J, Galt T, Bonniaud P, et al. Transfer of the active form of transforming growth factor-beta1 gene to newborn rat lung induces changes consistent with bronchopulmonary dysplasia. Am J Pathol 2003; 163: 2575-2584.

21 Zhao J, Sime PJ, Bringas P Jr, et al. Spatial-specific TGF-beta1 adenoviral expression determines morphogenetic phenotypes in embryonic mouse lung. Eur J Cell Biol 1999; 78: 715-725.

22 Zhao J, Bu D, Lee $\mathrm{M}$, et al. Abrogation of transforming growth factor-beta type II receptor stimulates embryonic mouse lung branching morphogenesis in culture. Dev Biol 1996; 180: 242-257.

23 Lecart C, Cayabyab R, Buckley S, et al. Bioactive transforming growth factor-beta in the lungs of extremely low birthweight neonates predicts the need for home oxygen supplementation. Biol Neonate 2000; 77: 217-223.

24 Choi CW, Kim BI, Joung KE, et al. Decreased expression of transforming growth factor-beta1 in bronchoalveolar lavage cells of preterm infants with maternal chorioamnionitis. J Korean Med Sci 2008; 23: 609-615.

25 Northway WH Jr, Rosan RC, Porter DY. Pulmonary disease following respirator therapy of hyaline-membrane disease. Bronchopulmonary dysplasia. N Engl J Med 1967; 276: 357-368.

26 Wilson MG, Mikity VG. A new form of respiratory disease in premature infants. AMA J Dis Child 1960; 99: 489-499.

27 Que J, Wilm B, Hasegawa H, et al. Mesothelium contributes to vascular smooth muscle and mesenchyme during lung development. Proc Natl Acad Sci USA 2008; 105: 16626-16630. 